\title{
Construction of a Realistic, Whole-Body, Three- Dimensional Equine Skeletal Model using Computed Tomography Data
}

\author{
Alexander K. K. Lee ${ }^{1}$, Elizabeth W. UhI ${ }^{2}$, Michelle L. Osborn ${ }^{1}$ \\ ${ }^{1}$ Department of Comparative Biomedical Sciences, Louisiana State University School of Veterinary Medicine ${ }^{2}$ Department of Pathology, University of \\ Georgia College of Veterinary Medicine
}

\section{Corresponding Author}

Michelle L. Osborn

mosborn@lsu.edu

\section{Date Published}

February 25, 2021

\section{Citation}

Lee, A.K.K., Uhl, E.W., Osborn, M.L. Construction of a Realistic, Whole-Body, ThreeDimensional Equine Skeletal Model using Computed Tomography Data. J. Vis. Exp. (168), e62276, doi:10.3791/62276 (2021).

DOI

$10.3791 / 62276$
URL

jove.com/video/62276

\section{Abstract}

Therapies based upon whole-body biomechanical assessments are successful for injury prevention and rehabilitation in human athletes. Similar approaches have rarely been used to study equine athletic injury. Degenerative osteoarthritis caused by mechanical stress can originate from chronic postural dysfunction, which, because the primary dysfunction is often distant from the site of tissue injury, is best identified through modeling whole-body biomechanics. To characterize whole-body equine kinematics, a realistic skeletal model of a horse was created from equine computed tomography (CT) data that can be used for functional anatomical and biomechanical modeling. Equine CT data were reconstructed into individual three-dimensional (3D) data sets (i.e., bones) using 3D visualization software and assembled into a complete 3D skeletal model. The model was then rigged and animated using 3D animation and modeling software. The resulting 3D skeletal model can be used to characterize equine postures associated with degenerative tissue changes as well as to identify postures that reduce mechanical stress at the sites of tissue injury. In addition, when animated into 4D, the model can be used to demonstrate unhealthy and healthy skeletal movements and can be used to develop preventative and rehabilitative individualized therapies for horses with degenerative lamenesses. Although the model will soon be available for download, it is currently in a format that requires access to the $3 \mathrm{D}$ animation and modeling software, which has quite a learning curve for new users. This protocol will guide users in (1) developing such a model for any organism of interest and (2) using this specific equine model for their own research questions.

\section{Introduction}

Chronic lameness in horses is often associated with progressive degenerative tissue lesions similar to those of osteoarthritis (OA), a major public health problem in humans ${ }^{1,2,3,4,5,6,7,8,9}$. In human medicine, because therapeutic approaches focused on treating specific lesions 
(e.g., pharmacotherapy and direct chondral repair) have mostly failed, pathomechanical forces are now recognized as the root cause of tissue damage in OA. Aberrant or pathomechanical forces impact both bone and cartilage cells directly, inducing the release of inflammatory mediators and progressive tissue degeneration ${ }^{9}$. These observations indicate that unless the causative mechanical forces are corrected, many chronic degenerative bone and joint diseases will continue to progress. Hence, the therapeutic focus in human medicine is shifting to approaches that "unload" the affected joints through targeted exercise ${ }^{10,11 .}$ However, this shift has not yet been made in equine medicine, partly because models for equine motion that can be adapted to show an individual's movements are needed.

Comprehensive, whole-body biomechanical analysis is common in designing training programs to optimize athletic performance and facilitate injury recovery in human athletes $^{11}$ (see also e.g., the journal "Sports Biomechanics"), but is less commonly done for equine athletes (but $\left.\mathrm{see}^{12}\right)$. Thus, the overarching goal here is to establish pathomechanical models of equine lameness that can be used to develop individualized preventative and rehabilitative therapies to improve the health of equine athletes. Such pathomechanical models can characterize differences in the functional anatomy of regions (i.e., the spine) that are not as easily discernible to the naked eye as others (i.e., the lower limb). To achieve this goal, the first objective was to develop an anatomically accurate, manipulatable, wholebody, equine skeletal model that can be used as a template by researchers interested in functional anatomical, kinematic, and kinetic analyses. To be useful to equine clinicians and researchers, this model must (1) be biologically realistic to enable accurate anatomical positioning, (2) allow for easy and accurate adjustments for modeling various postures of healthy and non-healthy horses, (3) be able to be animated to study the effects of various gaits, and (4) facilitate repeatable re-creations of positions and movements.

A 3D graphic whole-body equine skeletal model was built from CT data in which the positions of bones relative to each other could be manipulated and then animated to match movements from pictures or videos of a horse in motion, thus creating a 4D equine skeletal model. Depending upon what best fits the question to be addressed, the model can be used in $2 \mathrm{D}, 3 \mathrm{D}$, and $4 \mathrm{D}$ versions or in various combinations to illustrate and characterize the pathomechanical effects of specific positions or postures. Because of its basic and flexible design, the model serves as a template that can be modified by researchers to reflect their specific questions and data parameters. Such parameters include, for example, anatomical information based on sex and animal size, 3D motion analysis data, soft tissue force estimations, and inertial properties. Thus, the model allows for more detailed analysis of specific areas or joints, while also providing the basis to set up experiments that are unable to be performed on living horses. Due to practical limitations related to specimen availability (e.g., the ribs cut) and the scanner, the whole-body equine model is the result of merging data from three equine specimens. Thus, the model is not a perfect representation of a single individual, but has been standardized to represent individual variability more broadly. In short, it is a template to be used and modified to suit the needs of researchers. CT scans of the trunk, head and neck, and limbs were acquired from two equine specimens of approximately the same size with a 64-slice CT scanner using a bone algorithm, pitch of $0.9,1 \mathrm{~mm}$ slice. CT scans of a set of ribs were acquired with a 64-slice CT scanner using a bone algorithm, pitch of 0.9 , $0.64 \mathrm{~mm}$ slices. 
Anatomic integrity of the bony joints (e.g., within the limb) was maintained. The soft tissues available in the CT scans were also used to confirm the placement of the bones. As some whole ribs and the proximal portions of all the ribs were available and scanned on the thorax specimen, the separately scanned ribs could be accurately sized and placed within the whole-body skeletal model. The resulting CT Digital Imaging and Communications in Medicine (DICOM) data were imported into the $3 \mathrm{D}$ visualization software (see the Table of Materials), and individual bones were segmented into individual data sets (i.e., bone meshes). The individual $3 \mathrm{D}$ bone meshes were then imported into the $3 \mathrm{D}$ animation and modeling software (Table of Materials) where they were sized, if necessary, and assembled into a complete equine skeleton in preparation for rigging-a graphic method of connecting the bone meshes so that their movements are linked (Figure 1).

\section{Protocol}

\section{Forelimb rigging}

1. Place graphic joints inside the forelimb in all areas of movement.

NOTE: The resulting joint placement is a joint chain from the scapula to the distal end of the coffin bone (Figure 2A). In the area of the carpal bones, 3 joints in close proximity are used to increase the bending radius.

1. Press the $\mathbf{F} \mathbf{3}$ key to enable the Rigging Menu set. In the menus, select Skeleton | Create Joints to select the Create Joints tool.

2. In the View panel of the software, click in the approximate areas of the joints found in Figure 2A in the order of 1 to 10, and press the ENTER key.
3. Adjust the position of the joints by clicking on the desired joint and use the Move Tool by pressing the W key to translate the joint into the desired position. Alternatively, adjust a joint by clicking on the desired joint and altering the Translate $\mathbf{X}$, Translate $\mathbf{Y}$, and Translate $Z$ values found in the Channel Box/Layer Editor panel.

2. Create 5 separate Inverse Kinematic handles (IK handles) (joints will be referred to by the numbers found in Figure 2A).

1. In the menus, select Skeleton | Create IK Handle to select the Create IK Handle tool. Using the Create IK Handle tool, select joint 1, then joint 3; name this IK handle Front Leg IK in the Outliner panel. Using the Create IK Handle tool, select joint 3, then joint 7; name this IK handle Front Lower IK.

2. Using the Create IK Handle tool, select joint 7, then joint 8; name this IK handle Front Toe 1 IK in the Outliner panel. Using the Create IK Handle tool, select joint 8, then joint 9; name this IK handle Front Toe 2 IK in the Outliner panel. Using the Create IK Handle tool, select joint 9, then joint 10; name this IK handle Front Toe 3 IK in the Outliner panel.

3. Create forelimb controls

1. Create a Non-Uniform Rational B-Splines (NURBS) circle by using the Circle tool in the menu Create | NURBS Primitives | Circle.

2. Create two NURBS Circles and move them using the Move Tool to encircle joint 3 and joint 10, and name them Front Ctrl and Front Lower Ctrl, respectively, in the Outliner panel. 
3. Create a NURBS Circle; select the circle, and in the Channel Box/Layer Editor Panel, change the Rotate $\mathbf{Z}$ value to 90 . Using the Move tool, place it at the tip of joint 10, and name it Front Flick Ctrl in the Outliner panel.

4. Group Front Toe 1 IK, Front Toe 2 IK, and Front Toe $\mathbf{3}$ IK by selecting all three and pressing the CTRL + G keys. Name this group Front Toe Group in the Outliner panel. Parent the IK handles and Front Toe Group to the controls.

NOTE: It is important to Shift + select in the exact order described below to ensure a proper parent tree.

1. Select Front Leg IK, then Front Ctrl in the Outliner panel, and press the $\mathbf{P}$ key.

2. Select Front Lower Ctrl, then Front Ctrl in the Outliner panel, and press the $\mathbf{P}$ key.

3. Select Front Lower IK, then Front Lower Ctrl in the Outliner panel, and press the $\mathbf{P}$ key.

4. Select Front Flick Ctrl, then Front Lower Ctrl in the Outliner panel and press the $\mathbf{P}$ key.

5. Select Front Toe Group, then Front Flick Ctrl in the Outliner panel, and press the $\mathbf{P}$ key.

5. Use the Bind Skin tool to bind the bone meshes, except sesamoid bones, including navicular bones to the most proximal joint. Ensure that each bone mesh is only bound to one joint.

1. Click on the bone mesh, Shift + click on the most proximal joint, and select the Bind Skin tool under Skin | Bind Skin.

6. Rigging sesamoid bones and the navicular bone

1. Create a joint, place it in the middle of sesamoid bone, and press the Enter key. In the View panel, select the sesamoid bone mesh, and Shift + click the joint in the middle of the bone. Use the Bind Skin tool to bind the mesh to the joint.

NOTE: The sesamoid bone can now be manipulated using the Move and Rotate tools for adjustment when changing the leg position.

2. In the View panel, select the joint in the sesamoid bone, Shift + click the nearest joint in the forelimb, and press the $\mathbf{P}$ key.

NOTE: This parents the joint in the sesamoid bone to the forelimb.

3. Repeat steps 1.6 .1 to 1.6 .2 for other sesamoid bones and the navicular bone.

7. Repeat steps 1.1 through 1.6 for the other forelimb.

NOTE: The joint at the scapula can be selected and translated in all 3 directions (6 degrees of freedom) using the Move tool.

\section{Hindlimb rigging}

1. Place joints inside the hindlimb in all areas of movement to obtain a joint chain from the head of the demur to the distal end of the coffin bone (Figure 2B).

2. Create 5 separate IK handles (joints will be referred to the numbers found in Figure 2B).

1. Using the Create IK handle tool, select joint 11, then joint 12; name this IK handle Hind IK in the Outliner panel. Using the Create IK handle tool, select joint 12, then joint 14; name this IK handle Hind Lower IK in the Outliner panel.

2. Using the Create IK handle tool, select joint 14, then joint 15; name this IK handle Hind Toe $\mathbf{1}$ IK in the Outliner panel. Using the Create IK handle tool, 
select joint 15, then joint 16; name this IK handle Hind Toe 2 IK in the Outliner panel.

3. Using the Create IK handle tool, select joint 16, then joint 17; name this IK handle Hind Toe $\mathbf{3}$ IK in the Outliner panel.

3. Create hindlimb controls

1. Create two NURBS Circles named Hind Ctrl and Hind Lower Ctrl and move them to encircle joint 12 and joint 17 , respectively.

2. Create a NURBS Circle named Hind Flick Ctrl. Make this circle vertical, and place it at the tip of joint 10.

4. Group Hind Toe $\mathbf{1} \mathrm{IK}$, Hind Toe $2 \mathrm{IK}$, and Hind Toe $\mathbf{3}$ IK by selecting all three and pressing CTRL $+\mathbf{G}$. Name this group Hind Toe Group.

5. Parent the IK handles and Hind Toe Group to the controls. Be sure to Shift + select in the exact order described below to ensure a proper parent tree.

1. Select Hind IK, then Hind Ctrl, and press the P key.

2. Select Hind Lower Ctrl, then Hind Ctrl, and press the $\mathbf{P}$ key.

3. Select Hind Lower IK, then Hind Lower Ctrl, and press the $\mathbf{P}$ key.

4. Select Hind Flick Ctrl, then Hind Lower Ctrl, and press the $\mathbf{P}$ key.

5. Select Hind Toe Group, then Hind Flick Ctrl, and press the $\mathbf{P}$ key.

6. Use the Bind Skin tool to bind the bone meshes to the most proximal joint. Ensure that each bone mesh is bound to only one joint.
1. Click on the bone mesh, Shift + click the most proximal joint, and select the Bind Skin tool under Skin | Bind Skin.

7. Rigging patella, sesamoid bones, and navicular bone

1. Create a joint, place it in the middle of the patella, and press the Enter key. In the View panel, select the patella mesh, and Shift + click on the joint in the patella. Use the Bind Skin tool to bind the mesh to the joint.

NOTE: The patella can now be manipulated using the Move and Rotate tools for adjustment when changing the leg position.

2. In the View panel, select the joint in the patella, Shift + click on the nearest joint in the forelimb, and press the $\mathbf{P}$ key to parent the joint in the patella to the forelimb.

3. Repeat steps 2.7.1 and 2.7.2 for the sesamoid bones and the navicular bone.

8. Repeat steps 2.1 through 2.7 for the other hindlimb.

\section{Ribbon spine rigging}

1. Create a NURBS Plane with altered options with the length roughly equal to the length of the spine with $1 \mathrm{U}$ patch and \# V-patches, where \# is the number of thoracic and lumbar vertebrae.

NOTE: For this paper, the length is 20 with $22 \mathrm{~V}$ patches.

1. Select the square found next to the Create Plane tool under Create | NURBS Primitives | Plane.

2. Rebuild the plane with altered options.

1. Press the $\mathbf{F} \mathbf{2}$ key to enter the modeling menu set. Select the plane in the view panel, and select the Rebuild tool settings by selecting the square next to 
the Rebuild tool under Surfaces | Rebuild. Use the following options: number of spans $U=1$; number of spans $V=\#$ (22 in this case); select "1 Linear" for both the Degree $U$ and Degree $V$ options; keep the other settings to default; and press the Rebuild button.

3. Create nhairs with altered options.

1. Press the $\mathbf{F} \mathbf{5}$ key to enter the FX menu set. "Select the plane in the view panel, and use the Create Hair tool with altered options by selecting the square next to nHair | Create Hairs. Use the following options: output set to NURBS curves; $U$ count $=1 ; \mathrm{V}$ count = \# (22 in this case); keep the other options to default; and press the Create Hairs button.

4. Delete the following in the outliner panel: nucleus1, hairSystem1OutputCurves group, and hairSystem1. Fully expand the group labeled hairSystem1Follicles, and delete all the items labeled with curve

NOTE: The result should leave a group labeled hairSystem1Follicles that contains a list of items labeled nurbsPlane_Follicle

5. Select the plane, and move and orient it so that it is roughly overlapping with the spine by using the Move tool and Rotate tool. Select the plane, hold the right mouse button, and select Control Vertex to make all the vertices of the plane visible.

6. Move the vertices to orient the follicles to be between the vertebrae at the height where the spinal cord would be. Create \# number of separate joints (22 in this case) at any place in the View panel as the position of these joints will be corrected in later steps.

7. Parent a joint with a nurbsPlane_Follicle so that each has a single joint under its tree.
1. In the Outliner panel, select a joint created in step 3.6, then Ctrl + click on a nurbsPlane_Follicle___, and press the $\mathbf{P}$ key.

Repeat 3.7.1 with the other joints created in step 3.6 and the other nurbsPlane_Follicle objects.

8. In the Outliner panel, Ctrl + select all the joints; in the Chanel Box/Layer Box panel, set the Translate $X, Y$, and $\mathbf{Z}$ to $\mathbf{0}$. Duplicate all the joints by $\mathbf{C t r l}+$ selecting all the joints in the Outliner panel and pressing the Ctrl + D keys. Un-parent all the duplicate joints by Ctrl + selecting all the duplicate joints in the Outliner panel and pressing the Shift $+\mathbf{P}$ keys

9. Bind the joints under nurbsPlane_Follicle with their respective vertebra mesh.

1. Press the $\mathbf{F} \mathbf{3}$ key to enter the Rigging menu set. Click on the original joint (not the duplicate joint) under nurbsPlane_Follicle Shift + click on the respective vertebra mesh, and then use the Bind Skin tool under Skin | Bind Skin. Repeat these actions in step 3.9.1 for each joint and vertebrae mesh.

10. CTRL + click all duplicate joints and the plane, and use the Bind Skin tool to bind all the duplicate joints to the plane.

NOTE: The duplicate joints can now be manipulated to control the vertebrae.

11. Repeat steps 3.1 through 3.10 for the cervical and caudal vertebrae.

\section{Rib and sternum rigging}

1. Place separate joints at the rib head, at the proximal end of the costal cartilage, and at the distal end of the costal 
cartilage. Parent the joint at the proximal end of the costal cartilage to the joint at its rib head.

2. Parent the joint at the distal end of the costal cartilage to the closest joint at the proximal end of the costal cartilage. Parent the joint at the rib head to the spine joint that controls the vertebrae caudal to the rib.

3. In the Rigging menu set under the Skin tab, use the Bind Skin tool to bind the rib to the joint at its head and the costal cartilage to both the joints at its proximal end and the distal end.

4. Repeat steps 4.1 through 4.3 for each rib.

5. Place separate joints at the most cranial end of each sternal segment. Parent each sternal segment joint to the spinal joint most dorsal to each sternal segment joint. In the Rigging menu set under the Skin tab, use the Bind Skin tool to bind the sternal segment to its joint.

\section{Positioning and animation}

1. Select a frame in the timeline.

2. Position the model and controls. Import an image to use as a reference by creating a Free Image Plane.

NOTE: The images from Muybridge ${ }^{13}$ of the horse at the walk were used as proof of concept.

1. While the Free Image Plane is selected, select the image file under the Attribute Editor tab and under the Image Plane Attributes dropdown menu.
3. Select all controls and the spine control joints, and press the $\mathbf{S}$ key to save them as a key frame.

4. Along different frames along the timeline, move and rotate the controls and spine control joints, and press $\mathbf{S}$. NOTE: Repositioning controls and spine control joints and saving them as key frames along different points of the timeline creates an animation. There need not be a key frame set along each frame of the timeline; only critical positions or timings need to be key-framed. The 3D animation and modeling software will interpolate between the key-framed positions of each control and spine control joint, creating a smooth animation.

\section{Representative Results}

The result of the method was a 3D full equine skeletal model inside the 3D animation and modeling software that allows for accurate anatomical positioning and movement simulations. The model itself has a graphic rigging system delegated to the forelimbs, hindlimbs, spine, neck, and ribcage. The 3D model could be placed into different postures (Figure 3 and Figure 4) by multiple individuals. The movements of the 4D model (in motion) have been compared to videos from the side, back, and front, as well as with overhead drone footage to more accurately depict the motion of the spine and video of horses at the walk (Video), canter, and trot to create animations of those gaits. 


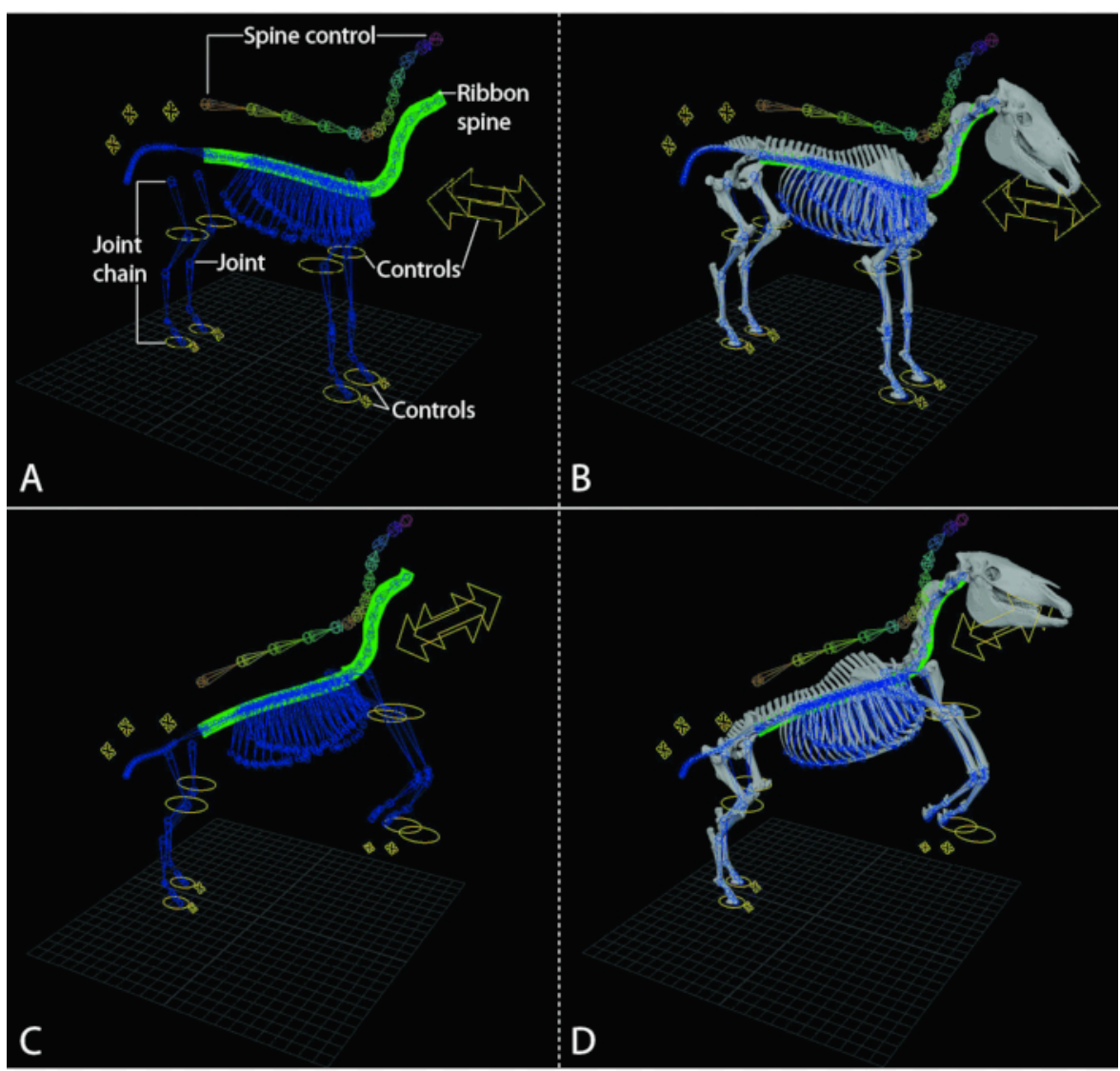

Figure 1: The 3D equine model can be moved into various postures and animated to demonstrate whole-body movements in various gaits in the 3D animation and modeling software. (A,C) Graphic rigging systems for the horse. The graphic ribbon spine that enables natural movement of the bony spine is illustrated by the green plane. The controls used to move the various graphic rigs and the attached bone meshes are illustrated by the yellow ovals and cross arrows on the model. (A) Standing position. (C) Rearing position. (B, D) The model with the bone meshes attached to the graphic rigging system. The positions of the controls change the position of the skeleton of the horse. (B) Standing horse. (D) Rearing horse. Please click here to view a larger version of this figure. 


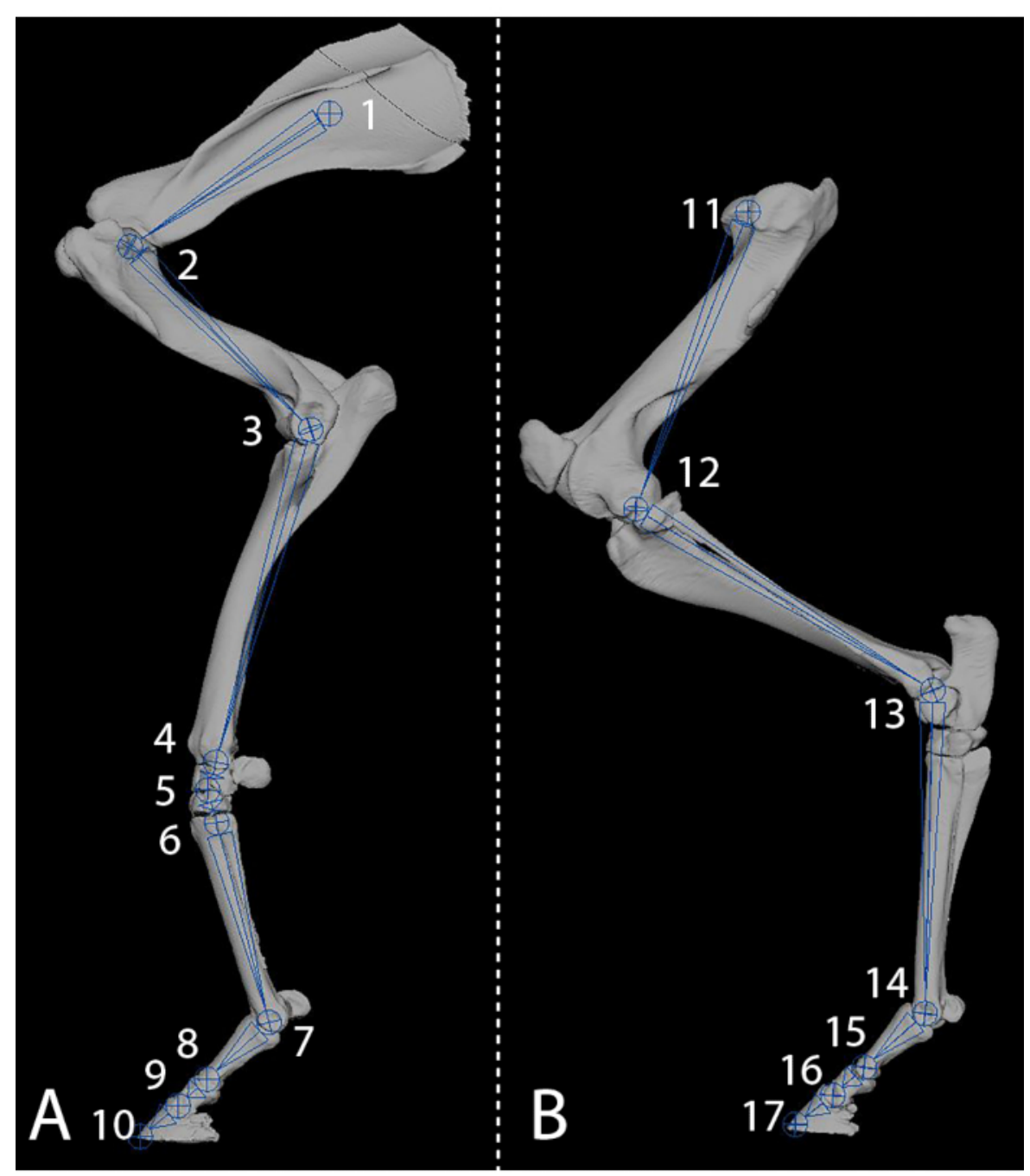

Figure 2: The rigging of each limb with joints allows for positioning and the creation of movement. (A) Forelimb with graphic joints indicated with numbers 1-10. (B) Hindlimb with graphic joints indicated with numbers 11-17. Please click here to view a larger version of this figure. 


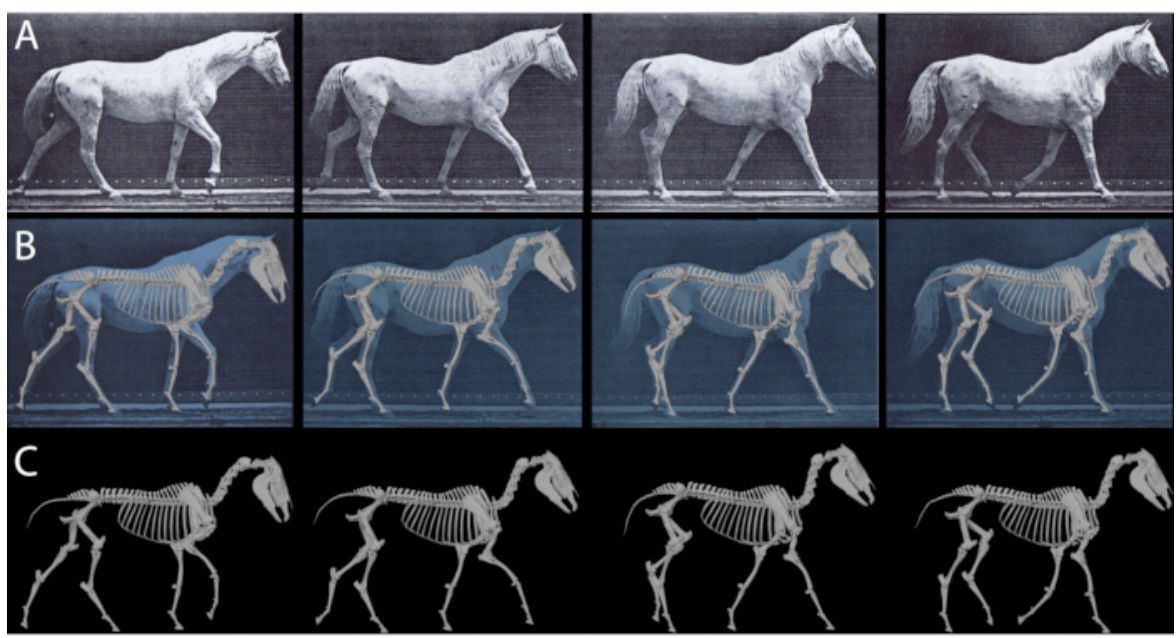

Figure 3: The 3D equine model was matched to classic Muybridge ${ }^{13}$ photos as proof of concept and to create the first animations. (A) Muybridge photographs of a horse at the walk. (B) The 3D equine model superimposed over the photographs to be used as key frames in the animation. (C) The 3D equine model. Please click here to view a larger version of this figure. 

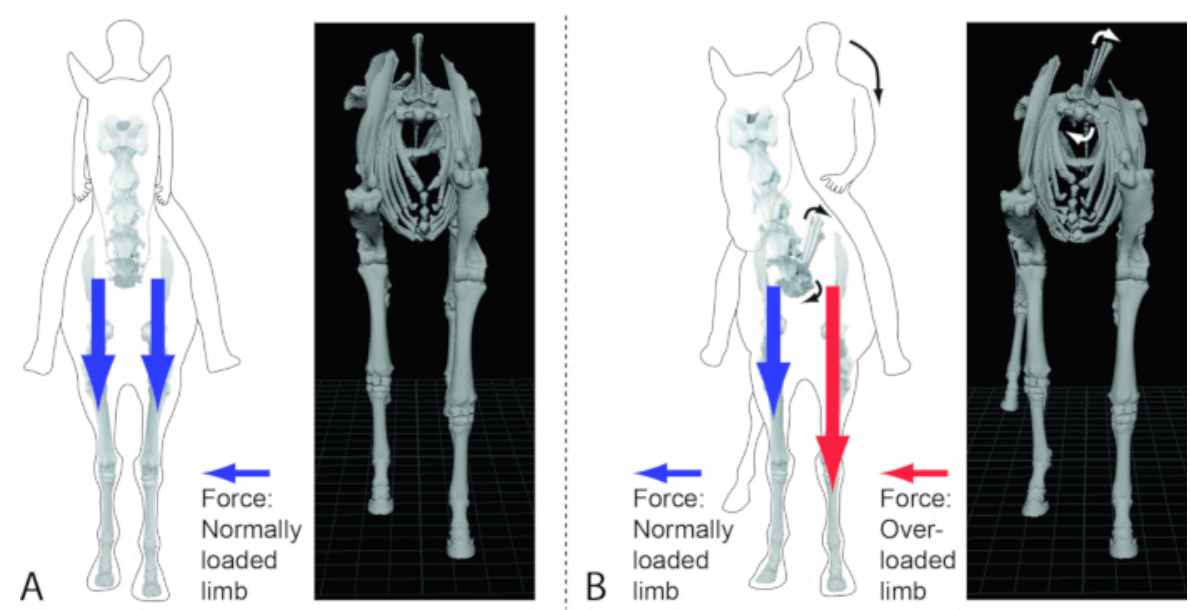

Figure 4: The 3D equine model can be moved into various postures (e.g., the transversal rotation of the spine demonstrated here) to understand the relationship of such postures to pathomechanical force regimes and the resulting degeneration of the affected skeletal elements, joints, and soft tissues. (A) A graphic $2 D$ representation of a normal posture of a horse (with rider) using graphically manipulated photographs of an equine skeleton compared to a still image of the 3D equine model with the head and cervical vertebrae hidden to enable the visualization of the thorax. (B) A graphic 2D representation of a horse (with rider) with a transversal rotation of the spine using graphically manipulated photographs of an equine skeleton compared to a still image of the 3D equine model with the head and cervical vertebrae hidden to enable the visualization of the thorax. Note here the effect of the transversal rotation on the skeleton and the limbs of the body. The depicted position would overload the left forelimb, which was supported by the compression and cracking of the left front hoof wall in the living horse. Please click here to view a larger version of this figure.

Video. The 4D Horse. Key positions of the skeleton, as matched to the Muybridge ${ }^{13}$ photos of the horse, have been interpolated to create an animation of the horse at a walk. The movement can be seen from the front, side, top, and back. Please click here to download this Video.

\section{Discussion}

This protocol demonstrates how to create a $3 D$ wholebody skeletal model of an organism and demonstrates how to use the whole-body equine skeletal model described in this paper. The model is currently in a format that requires a specific $3 \mathrm{D}$ animation and modeling software, which has quite a learning curve for new users. However, a version of this software is freely available for those who are affiliated with a university. Although modeling whole-body posture and movement is used to assess human athletes and to identify causes of mechanically induced chronic injuries ${ }^{11}$, it is less commonly done with equine athletes. To use this approach for the assessment of the potential causes of equine athletic injuries and performance issues, a realistic whole-body skeletal equine model was created from CT data using the 3D visualization software and 3D animation and modeling software. This model is different from other equine models that are either artistic graphic 
recreations of the skeleton (https://www.youtube.com/watch? $\mathrm{v}=$ YncZtLaZ6kQ) or that only depict the limbs ${ }^{14,15,16,17}$. In this whole-body model, forelimbs, hindlimbs, spine, and ribcage were all rigged and had controls attached that allow for easy manipulation of the model for realistic and accurate positioning and animation.

The protocol used to rig the model allows for repeatability and future alterations to suit the needs of the specific horse being rigged, enabling individualized analysis. Thus, the equine model is a tool to be used by researchers as they analyze movement. However, it is not an automated program that provides answers without the input of parameters specific to the animal being modeled and the question being addressed, as the accuracy of the model is directly related to the strength of a particular analysis. The ability to input parameters also allows the model to be continually updated with data from future research studies. Additionally, this graphic rigging protocol can be applied and/or adjusted to reflect the anatomical differences between individuals. It can also be adapted to effectively model other animals. The $3 \mathrm{D}$ equine model can be easily manipulated and positioned to simulate positions and movements. This is especially evident with the limbs as their movements are relatively simple to see and model.

Graphic joint positioning in the model was determined by a similar approach to that used in other studies ${ }^{18,19}$. The bone meshes were placed in a neutral position. Graphic joints were positioned so that the bones were able to rotate freely without causing any collision with other bone meshes. In the digits, the graphic joint was placed at the point where a sphere coincided with the surfaces of movement. The graphic joint of the scapula was placed in the approximate center of the scapula blade. This positioning of the graphic joint allows it to be moved in 6 degrees of freedom to orient the scapula into the desired position. Unlike the limbs, the movement of the spine is not easily seen, is more complex than often realized, and thus is more difficult to model. Although the model has the flexibility to be used to investigate movements and issues at specific spinal joints, it also needed to be able to represent the often hard-to-distinguish movements of the whole spine. The use of the "ribbon spine" allows for more realistic movement of the spine during animations.

This is important as the spine in horses, as has been found in humans, is often the origin of issues that are potentially related to aberrant biomechanical movements and injury to the limbs. A strength of this model is the ability to accurately demonstrate spine positions, like transversal vertebral rotations ${ }^{20}$ (Figure 4). How these postures impact the limbs in three dimensions during various gaits can be determined by using the model in combination with kinematic and force analysis (e.g., pressure plate studies to confirm increased loading of the limbs and static force analysis). Soft tissue musculofascial components are currently being added to the whole-body skeletal model. Future goals are to expand the use of the model in 3D biomechanical analysis for studies of equine lameness. Such expansion would include using the model to complete $3 \mathrm{D}$ force analyses that compare healthy and unhealthy postures and registering the model with 3D data points collected in motion capture studies to provide a more effective visual representation of movement.

\section{Disclosures}

The authors have declared no conflicts of interest. 


\section{Acknowledgments}

The authors acknowledge Mr. Jean Luc Cornille, Science of Motion, for his input into modeling accuracy; Dr. Martha Littlefield and Mr. James Ray (LSU SVM), and Dr. Steve Holladay, Dr. Carla Jarrett, and Mr. Brent Norwood (UGA CVM) for access to anatomical specimens; Dr. Ajay Sharma (UGACVM) and Dr. L. Abbigail Granger and Mr. Mark Hunter (LSUSVM) for conducting CT scans; and undergraduate researchers Jeremy Baker, Joshua Maciejewski, Sarah Langlois and Daniel Pazooki (LSU School of Veterinary Medicine Functional and Evolutionary Anatomy Lab) for their work related to this research. Funding was procured from the Louisiana State University School of Veterinary Medicine's Equine Health Studies Program via a Charles V. Cusimano grant.

\section{References}

1. Ostblom, L., Lund, C., Melsen, F. Histological study of navicular bone disease. Equine Veterinary Journal. 14 (3),199-202 (1982).

2. Rossdale, P. D., Hopes, R., Digby, N. J. W., Offord, K. Epidemiological study of wastage among racehorses 1982 and 1983. The Veterinary Record. 116 (3), 66-69 (1985).

3. Pool, R .R., Meagher, D. M., Stover, S. M. Pathophysiology of navicular syndrome. Veterinary Clinics of North America: Equine Practice. 5 (1), 109-129 (1989).

4. Blunden, A., Dyson, S., Murray, R., Schramme, M. Histopathology in horses with chronic palmar foot pain and age-matched controls. Part 1: Navicular bone and related structures. Equine Veterinary Journal. 38 (1), $15-22(2006)$.

5. Dyson, S., Murray, R. Magnetic resonance imaging evaluation of 264 horses with foot pain: the podotrochlear apparatus, deep digital flexor tendon and collateral ligaments of the interphalangeal joint. Equine Veterinary Joint. 39 (4), 340-343 (2007).

6. Dyson, S., Murray, R. Use of concurrent scintigraphic and magnetic resonance imaging evaluation to improve understanding of the pathogenesis of injury of the podotrochlear apparatus. Equine Veterinary Journal. 39 (4), 365-369 (2007).

7. Egenvall, A., Lonnell, C., Roepstorff, L. Analysis of morbidity and mortality data in riding school horses, with special regard to locomotor problems. Preventive Veterinary Medicine. 88 (3), 193-204 (2009).

8. Waguespack, R., Hanson, R. R. Navicular syndrome in equine patients anatomy, causes, and diagnosis. Compendium: Continuing Education for Veterinarians. 32 (12), E1-E14 (2010).

9. Zhen, G., Cao, X. Targeting TGF $\beta$ signaling in subchondral bone and articular cartilage homeostasis. Trends in Pharmacological Sciences. 35 (5), 227-236 (2014).

10. Arendt, E. A., Miller, L. E., Block, J. E. Early knee osteoarthritis management should first address mechanical joint overload. Orthopedic Reviews. 6 (1), 5188 (2014).

11. Rietveld, A. B. Dancers' and musicians' injuries. Clinical Rheumatology. 32 (4), 425-434 (2013).

12. Parkes, R., Newton, R., Dyson, S. Is there an association between clinical features, response to diagnostic 
analgesia and radiological findings in horses with a magnetic resonance imaging diagnosis of navicular disease or other injuries of the podotrochlear apparatus? Veterinary Journal. 204 (1), 40-46 (2015).

13. Muybridge, E. Animals in motion. Chapman \& Hall, London (1902).

14. Brown, N. A. T., Pandy, M. G., Kawcak, C. E., Mcllwraith, C. W. Force- and moment-generating capacities of muscles in the distal limb of the horse. Journal of Anatomy. 203 (1), 101-113 (2003).

15. Harrison, S. M., Whitton, R. C., Kawcak, C. E., Stover, S. M., Pandy, M. G. Relationship between muscle forces, joint loading and utilization of elastic strain energy in equine locomotion. Journal of Experimental Biology. 213 (23), 3998-4009 (2010).

16. Harrison, S. M. et al. Forelimb muscle activity during equine locomotion. Journal of Experimental Biology. 215 (17), 2980-2991 (2012).

17. O'Hare, L. M. S., Cox, P. G., Jeffery, N., Singer, E. R. Finite element analysis of stress in the equine proximal phalanx. Equine Veterinary Journal. 45 (3), 273-277 (2013).

18. Panagiotopoulou, O., Rankin, J., Gatesy, S., Hutchinson, S. A preliminary case study of the effect of shoe-wearing on the biomechanics of a horse's foot. PeerJ. 4, e2164 (2016).

19. Craig, J., Craig, M., Savoldi, M., Waldsmith, J. Locating rotation centers of the equine digit and their use in quantifying conformation. EponaMind. https://www.eponamind.com/blog/locatingrotation-centers-of-the-equine-digit-and-their-use-inquantifying-conformation/ (2005).
20. Denoix, J. M. Spinal biomechanics and functional anatomy. Veterinary Clinics of North America: Equine Practice. 15 (1), 27-60 (1999). 